\title{
Hydrophobe-substituted bPEI derivatives: boosting transfection on primary vascular cells
}

\author{
Daniele Pezzoli $^{1 *}$, Eleni K. Tsekoura ${ }^{2}$, Remant Bahadur K.C. ${ }^{2}$, Gabriele Candiani ${ }^{3}$, Diego Mantovani ${ }^{1}$ \\ and Hasan Uludağ ${ }^{2 *}$
}

\begin{abstract}
Gene therapy targeted to vascular cells represents a promising approach for prevention and treatment of pathological conditions such as intimal hyperplasia, in-stent and post-angioplasty restenosis. In this context, polymeric non-viral gene delivery systems are a safe alternative to viral vectors but a further improvement in efficiency and cytocompatibility is needed to improve their clinical success. Herein, a library of 24 branched polyethylenimine (bPEI) derivatives modified with hydrophobic moieties was synthesised, characterised and tested in vitro on primary vascular cells, aiming to identify delivery agents with superior transfection efficiency and low cytotoxicity. Low molecular weight PEIs (0.6, 1.2 and $2 \mathrm{kDa}$ ) were grafted with long $(\mathrm{C18})$ and short (C3) aliphatic chains, featuring different unsaturation degrees and degrees of substitution. $0.6 \mathrm{kDa} b$ PEI-based derivatives were generally ineffective in transfection on vascular smooth muscle cells (VSMCs), while among the other derivatives some promising vectors were identified. Forcing polyplexes on the cell surface by means of centrifugation invariably boosted transfection levels but increased cytotoxicity as well. Of note, a propionyl-substituted derivative (PEI2-PrA1, C3:0) was the most effective on both VSMCs and endothelial cells (ECs), with higher and more sustained gene expression in combination with markedly lower cytotoxicity with respect to the gold standard $25 \mathrm{kDa} b$ PEI. In addition, a linoleoyl-substituted derivative (PEI1.2-LA6, C18:2) owing to its high efficiency in VSMCs and relative inefficacy in ECs, combined with tolerable cytotoxicity was proposed as a vector for specific VSMCs targeting.
\end{abstract}

Keywords: non-viral gene delivery, polyethylenimine, hydrophobic substitution, smooth muscle cells, endothelial cells, transfection

\section{INTRODUCTION}

Cardiovascular diseases are the leading cause of death worldwide [1]. Consequently, vascular interventions represent one of the most common surgical procedures, whose numbers are predicted to further increase owing to the growth of the elderly demographic of the population [2]. Proliferation, migration and phenotype switch of vascular smooth muscle cells (VSMCs) play pivotal roles in several pathological vascular conditions such as intimal hyperplasia and are crucial events leading to in-stent and post-angioplasty restenosis [3]. In addition, endothelial cells (ECs) are strongly involved in these phenomena since they are responsible for endothelial regeneration and can regulate VSMC proliferation and phenotypic switch [4]. In this context, gene therapy targeted to VSMCs and ECs is a promising therapeutic approach for the targeted inhibition of mechanisms leading to the development of vascular pathologies or to post-intervention complications [5]. Furthermore, in vitro gene delivery to primary VSMCs and ECs can be employed for tissue engineering and regeneration applications [6], and in the study of gene function and of the general physiology of vascular tissues [7].

Gene therapy involves the therapeutic introduction of DNA or RNA into cells with the goal of modifying their protein expression profile and halting disease progression. However, the efficient and safe delivery of nucleic acids to cells is still a key challenge towards the clinical application of gene therapy. Traditional gene delivery techniques used in laboratory are in fact difficult to effectively translate to in vivo [8]. Viral vectors are generally considered to be

\footnotetext{
${ }^{1}$ Laboratory for Biomaterials and Bioengineering, CRC- I, Department of Mining, Metallurgical and Materials Engineering and CHU de Québec Research Centre, Laval University, Quebec City, QC, G1V 0A6, Canada

${ }^{2}$ Department of Chemical \& Material Engineering, Faculty of Engineering, University of Alberta, Edmonton, AB, T6G 1H9, Canada

${ }^{3}$ Department of Chemistry, Materials and Chemical Engineering "Giulio Natta", Politecnico di Milano, Milan 20131, Italy

*Corresponding authors (emails: daniele.pezzoli.1@ulaval.ca (Pezzoli D); huludag@ualberta.ca (Uludağ H))
} 
the most efficient gene delivery systems, but their use is limited by the immune response exerted by the body and other major safety concerns. Chemical non-viral vectors represent a safer alternative, but further improvement of their efficiency, especially in primary cells, is still a key point to clinical success. Among non-viral vectors, cationic polymers display distinct advantages in terms of reproducibility, high degree of molecular diversity and of easiness of chemical functionalization that allows fine-tuning of their physicochemical properties and performance [9]. When exposed to polyanionic DNA, cationic polymers self-assemble into positively charged polymer/DNA complexes, named polyplexes, that protect nucleic acids from degradation and mediate their cellular uptake. Polyethyleneimine (PEI) is recognised as the gold standard polymeric gene carrier; it features a high charge density and a strong buffering capacity at acidic $\mathrm{pH}$ that elicits endosomal escape of PEI-based polyplexes via a process named "proton sponge effect" [10]. It is worth to note that high molecular weight (MW) PEIs display not only higher transfection efficiency but also greater cytotoxicity with respect to their low MW counterparts. To improve its efficacy and cytocompatibility, PEI has been chemically tailored with a number of different substituents $[9,11,12]$, among which, hydrophobic modification has been proven as a valuable approach. In fact, the grafting of hydrophobic chains on PEI results in increased cellular uptake via endocytosis and improved interactions between polyplexes and cell membranes [13]. These strategies mainly rely on the functionalization of cytocompatible low MW PEI with hydrophobic moieties to improve transfection efficiency, while retaining limited cytotoxicity. A number of different substitutes have been successfully grafted to PEI thus far: cholesterol [14], cholic acid, long-chain dodecyl and hexadecyl moieties [15], lipoic acid [16], saturated and unsaturated lipids of different lengths such as palmitic acid, stearic acid, oleic acid, and linoleic acid [13,17,18] and, recently, the small hydrophobic propionic acid [19].

In light of the promising results about hydrophobic moiety-substituted PEI and given the importance of developing safe and effective vectors for the transfection of VSMCs and ECs, in this study we explored and compared the potential of a library of hydrophobe-substituted branched PEI ( $b$ PEI) derivatives to transfect these two cell types. Low MW $b$ PEIs $(0.6,1.2$ and $2 \mathrm{kDa})$ were employed as backbone for the synthesis of the derivatives and five hydrophobic substituents were grafted at different grafting degrees aiming to finally identify the best transfection reagents and possibly draw structure-activity relationships.

\section{EXPERIMENTAL SECTION}

\section{Materials}

The plasmid DNA (pDNA) pGL3-Control Vector (5.2 $\mathrm{kb}$ ), encoding for the modified firefly luciferase and the Luciferase Assay System were purchased from Promega (Madison, WI, USA); the pDNA pGLuc-Basic 2 Vector, encoding for the secreted Gaussia luciferase and the BioLux Gaussia Luciferase Assay Kit were from New England Biolabs (Ipswich, MA, USA). Bicinchoninic acid (BCA) protein assay kit, Dulbecco's modified Eagle's medium (DMEM), M199 medium, Gibco Opti-MEM I Reduced Serum Medium, fetal bovine serum (FBS), penicillin/streptomycin solution $\left(100 \mathrm{U} \mathrm{mL}^{-1}\right.$ penicillin, $0.1 \mathrm{mg} \mathrm{\textrm {mL } ^ { - 1 }}$ streptomycin), phosphate-buffered saline solution (PBS), AlamarBlue Cell Viability Reagent, were from Thermo Fisher Scientific (Waltham, MA, USA). $2 \mathrm{kDa} b$ PEI $\left(M_{\mathrm{n}}\right.$ : $\left.1.8 \mathrm{kDa}, M_{\mathrm{w}}: 2 \mathrm{kDa}\right), 25 \mathrm{kDa} b$ PEI $\left(M_{\mathrm{n}}: 10 \mathrm{kDa}, M_{\mathrm{w}}\right.$ : $25 \mathrm{kDa}$ ), dimethyl sulfoxide (DMSO), stearoyl chloride (St), propionic acid ( $\mathrm{PrA}$ ) and acryloyl chloride ( $\mathrm{AoCl})$, 1-ethyl-3-(3-dimethylaminopropyl) carbodiimide (EDC), $\mathrm{N}$-hydroxysuccinimide (NHS), chloroform $\left(\mathrm{CHCl}_{3}\right)$, methanol $(\mathrm{MeOH})$ were obtained from Sigma-Aldrich (St. Louis, MO, USA). $0.6 \mathrm{kDa} b$ PEI and $1.2 \mathrm{kDa} b$ PEI were obtained from the Polysciences Inc. (Warrington, PA, USA). Linoleyl chloride (LA) and $\alpha$-linoleoyl chloride ( $\alpha$ LA) were obtained from NU-CHEK PREP (Elysian, MN, USA). Fluorimetric, spectrophotometric and luminescence analyses were performed using the multi-mode microplate reader system SpectraMax i3x (Molecular Devices, Sunnyvale, CA, USA).

\section{Polymer synthesis and characterization}

Hydrophobe-modified $b$ PEI derivatives were prepared by grafting hydrophobic molecules onto $b$ PEIs via $N$-acetylation as described earlier [17]. In a typical experiment, a lipid chloride $\left(2 \mathrm{mmol} \mathrm{L}^{-1}\right)$ and PEI $\left(1 \mathrm{mmol} \mathrm{L}^{-1}\right)$ were dissolved separately in ice-cold anhydrous chloroform for $30 \mathrm{~min}$. Triethylamine $(100 \mu \mathrm{L})$ was added to the PEI solution and cooled for another $30 \mathrm{~min}$ in ice bath. The lipid chloride solution was added dropwise to the PEI solution while being kept on ice bath under stirring. Reaction mixture was then left stirring overnight $(\sim 12$ h) at room temperature. Derivatives with propionic acid (PrA) and acryloyl chloride (AoCl) were prepared using EDC/NHS activation method as described in our earlier publication [19]. The crude product was precipitated 3 times in ice-cold diethylether and dried under vacuum for 2 days. The structural composition of the polymers 
was analyzed by ${ }^{1} \mathrm{H}-\mathrm{NMR}$ spectroscopy (Bruker $300 \mathrm{MHz}$, Billerica, MA, USA) using tetramethylsilane as an internal standard in $\mathrm{D}_{2} \mathrm{O}$ to calculate the lipid substitution levels, which are summarized in Table 1.

\section{Preparation of polyplexes}

Polyplexes were prepared at room temperature by adding an aqueous solution of pDNA $\left(0.4 \mu \mathrm{g} \mu \mathrm{L}^{-1}\right)$ to the $b \mathrm{PEI}$ derivatives diluted in serum-free medium (Opti-MEM) at the desired concentration (polymer solution), to obtain different polymer/pDNA $w / w$ ratios. The final pDNA concentration in the polyplex suspensions was $25 \mathrm{ng} \mu \mathrm{L}^{-1}$. Polyplex suspensions were incubated for $30 \mathrm{~min}$ at room temperature prior to use in transfection studies.

\section{DNA binding by polymers}

The ability of $b$ PEI derivatives to bind and complex DNA was evaluated by a fluorophore-exclusion assay using SYBR Green I $\left(\lambda_{\mathrm{ex}}=497 \mathrm{~nm} ; \lambda_{\mathrm{em}}=520 \mathrm{~nm}\right)$ as DNA dye [20]. Polyplexes (400 ng of pDNA per condition) were prepared in serum-free medium at different $w / w$ ratios as described above. Polyplexes were next diluted to $100 \mu \mathrm{L}$ in $10 \mathrm{mmol}$ $\mathrm{L}^{-1}$ HEPES ( $\mathrm{pH} 7.0$ ) containing $2 \times$ DNA dye and, after further 20 min incubation at room temperature, fluorescence was measured in a black 96-well microplate. The fluorescence of the samples was normalized over the signal of free pDNA. For each polymer, fluorescence $\%$ was plotted against polymer/pDNA $w / w$ ratio and fitted to a sigmoidal curve that was used to calculate the $\mathrm{BC}_{50}(w / w$ ratio requi-

Table 1 Properties of the library of $b$ PEI derivatives and of unmodified $b$ PEIs investigated in this study. The table summarizes the type of substitute, the lipid:PEI feed ratio $(\mathrm{mol} / \mathrm{mol})$ used during the reaction, the degree of substitution calculated from ${ }^{1} \mathrm{H}$ NMR analysis and the $w / w$ ratio required for $50 \%$ pDNA binding during complexation $\left(\mathrm{BC}_{50}\right)$, evaluated by SYBR Green I fluorophore-exclusion assay.

\begin{tabular}{|c|c|c|c|c|}
\hline Polymer & Substitute & Feed ratio $(\mathrm{mol} / \mathrm{mol})$ & Degree of substitution $(\mathrm{mol} / \mathrm{mol})$ & $\mathrm{BC}_{50}$ \\
\hline PEI2-St6 & Stearic acid & 6.0 & 2.14 & 0.678 \\
\hline PEI2-St12 & Stearic acid & 12.0 & 4.53 & 8.088 \\
\hline PEI0.6-LA4 & Linoleic acid & 4.0 & 1.09 & 0.364 \\
\hline PEI1.2-LA4 & Linoleic acid & 4.0 & 1.84 & 0.761 \\
\hline PEI1.2-LA6 & Linoleic acid & 6.0 & 2.55 & 0.686 \\
\hline PEI2-LA6 & Linoleic acid & 6.0 & 2.31 & 0.785 \\
\hline PEI2-LA9 & Linoleic acid & 9.0 & 3.20 & 3.609 \\
\hline PEI0.6- $\alpha$ LA2 & a-linoleic acid & 2.0 & 0.80 & 0.320 \\
\hline PEI0.6- $\alpha \mathrm{LA} 4$ & a-linoleic acid & 4.0 & 2.30 & 1.269 \\
\hline PEI1.2- $\alpha$ LA2 & a-linoleic acid & 2.0 & 0.94 & 0.289 \\
\hline PEI1.2-aLA4 & a-linoleic acid & 4.0 & 2.45 & 0.297 \\
\hline PEI1.2- $\alpha$ LA6 & a-linoleic acid & 6.0 & 3.17 & 0.578 \\
\hline PEI2- $\alpha \mathrm{LA} 2$ & a-linoleic acid & 2.0 & 1.37 & 0.681 \\
\hline PEI2- $\alpha$ LA4 & a-linoleic acid & 4.0 & 2.72 & 1.015 \\
\hline PEI2- $\alpha$ LA 8 & a-linoleic acid & 8.0 & 3.68 & 3.899 \\
\hline PEI0.6-PrA1 & Propionic acid & 1.0 & 0.62 & 0.298 \\
\hline PEI1.2-PrA0.5 & Propionic acid & 0.5 & 0.28 & 0.316 \\
\hline PEI1.2-PrA1 & Propionic acid & 1.0 & 0.76 & 0.310 \\
\hline PEI2-PrA0.5 & Propionic acid & 0.5 & 0.15 & 0.304 \\
\hline PEI2-PrA1 & Propionic acid & 1.0 & 0.53 & 0.367 \\
\hline PEI1.2-AcA1 & Acrylic acid & 1.0 & 0.65 & 0.343 \\
\hline PEI1.2-AcA2 & Acrylic acid & 2.0 & 1.21 & 0.430 \\
\hline PEI2-AcA1 & Acrylic acid & 1.0 & 0.51 & 0.355 \\
\hline PEI2-AcA2 & Acrylic acid & 2.0 & 0.86 & 0.643 \\
\hline $0.6 \mathrm{kDa} b \mathrm{PEI}$ & I & I & I & 0.278 \\
\hline $1.2 \mathrm{kDa} b \mathrm{PEI}$ & I & I & I & 0.215 \\
\hline $2 \mathrm{kDa} b \mathrm{PEI}$ & l & l & I & 0.213 \\
\hline $25 \mathrm{kDa} b \mathrm{PEI}$ & l & l & l & 0.274 \\
\hline
\end{tabular}


red for 50\% pDNA binding).

\section{Size and $\zeta$-potential of polyplexes}

The hydrodynamic diameter $\left(D_{\mathrm{H}}\right)$ and the $\zeta$-potential $\left(\zeta_{\mathrm{p}}\right)$ of $b$ PEI derivatives/pDNA complexes were measured through dynamic light scattering (DLS) and laser Doppler micro-electrophoresis using a Malvern Zetasizer Nano-ZS (Malvern Instruments, Malvern, UK) equipped with $\mathrm{He}-\mathrm{Ne}$ laser and operated at $10 \mathrm{~mW}$. Polyplexes were prepared at different $w / w$ as described above and measured after equilibration at $25^{\circ} \mathrm{C}$.

\section{Cells and cell culture}

Primary Porcine Aortic SMCs (PAoSMCs) were derived from tunica media of healthy, fibrous plaque-free porcine arteries. Briefly, PAoSMCs were isolated from $1 \mathrm{~cm}$ wide aortic rings cut from the aorta of a pig. Aortic segments were collected in PBS, placed in M199 medium supplemented with $1 \%$ penicillin/streptomycin solution and immediately dissected. The adventitia tissue and the first external third of the media were carefully removed and the resulting rings were cut in small pieces of approximately $1 \mathrm{~mm} \times 5 \mathrm{~mm}$ using a scalpel and moved into a Petri dish containing M199 medium supplemented with 10\% FBS and $1 \%$ penicillin/streptomycin solution (complete M199, hereafter referred to as cM199). Medium was changed every other day. The explants were removed after one week when a sufficient amount of SMCs was present on the surface of the Petri dish. PAoSMCs were then cultured in DMEM supplemented with $10 \%$ FBS and $1 \%$ penicillin/streptomycin solution (complete DMEM, hereafter referred to as $\mathrm{CDMEM}$ ) at $37^{\circ} \mathrm{C}$ in a humidified atmosphere of $5 \% \mathrm{CO}_{2}$.

Primary Human Umbilical Artery SMCs (HUASMCs) were purchased from Lonza (CC-2579, Walkersville, MD, USA) and cultured at $37^{\circ} \mathrm{C}$ in a humidified atmosphere under constant supply of $5 \% \mathrm{CO}_{2}$, in cDMEM enriched with $5 \mu \mathrm{g} \mathrm{mL}{ }^{-1}$ human insulin (Santa Cruz Biotechnology, Dallas, TX), $2 \mathrm{ng} \mathrm{mL}^{-1}$ fibroblast growth factor-basic (FGFb, Gibco by Life Technologies, Grand Island, NY, USA), and $0.5 \mathrm{ng} \mathrm{mL}^{-1}$ epidermal growth factor (EGF, Invitrogen by Life Technologies), hereafter referred to as CDMEM+.

Primary Human Umbilical Vein Endothelial Cells (HUVECs) were isolated from $\sim 15 \mathrm{~cm}$-long umbilical cord pieces obtained from normal-term pregnancies with the consent of the donor mothers. Briefly, the vein was rinsed with PBS, filled with $10 \times$ trypsin-EDTA solution (Gibco by Life Technologies) and incubated for $15 \mathrm{~min}$ at $37^{\circ} \mathrm{C}$. Then the trypsin-EDTA solution containing cells was collected,
PBS was added to wash the lumen of the vein, collected and added to the cell-containing suspension. The resulting cell suspension was centrifuged at $1,000 \mathrm{rpm}$ for $5 \mathrm{~min}$, the supernatant was removed and cells were resuspended in cM199 and seeded in a T75 flask. Culture medium was changed after $24 \mathrm{~h}$ and then every $48 \mathrm{~h}$ until confluence. ECs were cultured in cM199 supplemented with $2 \mathrm{ng}$ $\mathrm{mL}^{-1} \mathrm{FGFb}, 0.5 \mathrm{ng} \mathrm{mL} \mathrm{m}^{-1}$ EGF, $1 \mu \mathrm{g} \mathrm{mL} \mathrm{m}^{-1} \mathrm{~L}$-ascorbic acid

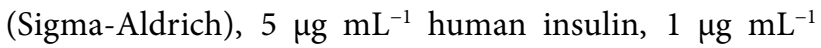
hydrocortisone (Sigma-Aldrich) and $90 \mu \mathrm{g} \mathrm{mL}^{-1}$ porcine heparin sodium salt Grade I-A (Sigma-Aldrich), hereafter referred to as cM199+.

The protocols for the isolation of PAoSMCs and HUVECs were approved by the Ethics Committee of CHU de Québec Research Centre. For all the experiments cells were used at passage between 6 and 10 .

\section{In vitro transfection experiments}

Twenty-four hours before transfection experiments PAoSMCs, HUASMCs and HUVECs were seeded in 96-well cell culture plates at a density of $2.0 \times 10^{4} \mathrm{cells} / \mathrm{cm}^{2}$ in the corresponding culture medium (cDMEM, cDMEM+ and cM199+, respectively). Twenty-four hours post-seeding, $160 \mathrm{ng} /$ well (PAoSMCs) or $80 \mathrm{ng} /$ well of pDNA (HUASMCs and HUVECs) were complexed with $b$ PEI derivatives at the desired $w / w$ and added to cells in fresh complete medium. The final pDNA concentration in the culture medium was $1 \mu \mathrm{g} \mathrm{mL} \mathrm{m}^{-1}$. Four hours after the addition of polyplexes, the transfection medium was replaced with $100 \mu \mathrm{L}$ of fresh culture medium. When indicated, cells were centrifuged at $500 \times \mathrm{g}$ for $5 \mathrm{~min}$ immediately after the addition of polyplexes.

\section{Evaluation of cytotoxicity and transfection efficiency}

The cytotoxicity of polyplexes was assessed by AlamarBlue cell viability assay. The medium was harvested and replaced with $100 \mu \mathrm{L}$ of complete medium containing $10 \%$ AlamarBlue solution. Cells were incubated in standard culture conditions for $3 \mathrm{~h}$ and the fluorescence of the medium $\left(\lambda_{\mathrm{ex}}=560 \mathrm{~nm} ; \lambda_{\mathrm{em}}=590 \mathrm{~nm}\right)$ was read using the SpectraMax i3x microplate reader. Viability of non-treated control cells was assigned to as $100 \%$ and cytotoxicity was determined as follows:

$$
\text { Cytotoxicity [\%] }=100 \%-\text { Viability [\%]. }
$$

The expression of firefly luciferase (pGL3 plasmid) was evaluated $48 \mathrm{~h}$ post-transfection by measuring the luciferase activity using the Luciferase Assay System as previously described [21]. Briefly, cells were washed with PBS, lysed with $0.25 \times$ Cell Culture Lysis Reagent (Promega) and 
freeze-thawed once. Then, $20 \mu \mathrm{L}$ of cell lysate were mixed with $50 \mu \mathrm{L}$ of Luciferase Assay Reagent and luminescence was immediately recorded by the microplate reader. The chemiluminescence signal of the samples was normalized to their corresponding protein content, determined by BCA assay. Data were finally expressed as relative light units per mg of proteins (RLU/mg of proteins).

The expression of Gaussia luciferase (pGLuc plasmid), secreted in the culture medium, was assessed at different time points by BioLux Gaussia Luciferase Assay Kit, according to manufacturer's instructions. Culture medium was harvested $24 \mathrm{~h}$ post-transfection or $24 \mathrm{~h}$ after every medium change and $20 \mu \mathrm{L}$ of medium were mixed with 50 $\mu \mathrm{L}$ of GLuc assay solution and luminescence was promptly measured by the microplate reader.

\section{Statistical analysis}

Results were expressed as mean \pm standard deviation. Statistical analyses were carried out by means of GraphPad Prism v 6.01 (GraphPad software, La Jolla, CA, USA). Comparisons among groups were performed by one-way analysis of variance (ANOVA) with Sidak-Bonferroni adjustment for multiple testing. Correlations were evaluated by Pearson correlation test. Significance was retained when $p<0.05$.

\section{RESULTS AND DISCUSSION}

Primary vascular cells, SMCs and ECs, as many other primary cells, are recognised difficult-to-transfect cell types [22-24]. In this study we investigated the efficiency of hydrophobe-substituted $b$ PEI derivatives in the transfection of these cells. Different fatty acid-based substitutes have been investigated, namely stearic acid (St, C18 chain), linoleic acid (LA, C18:2 $(6,9)$ chain), and propionic acid (PrA, C3 chain), already reported to improve the transfection activity of $b$ PEI on other cell types such as human embryonic kidney cells (293T) [17], rat bone marrow stromal cells (rBMSC) [25] and human breast cancer cells (MDA-231 and MCF-7) [19]. Besides, two new families of $b$ PEI derivatives, carrying $\alpha$-linoleic acid ( $\alpha$ LA, C18:3 $(3,6,9)$ chain) and acrylic acid (AcA, C3:1 (1) chain), were synthesised and studied, aiming to evaluate whether an increase in the level of unsaturation in the aliphatic chain of linoleoyl- and propionyl-based substitutes (i.e., an additional double bond, in position 3 and 1, respectively) could lead to a further enhancement in the transfection efficiency of $b$ PEI derivatives on vascular cells. Of note, to find out the optimal chemical structures and to draw overall structure-activity relationships, different $b$ PEI backbone MWs and degrees of substitution were tested.

\section{Synthesis and characterization of hydrophobe-substituted $b$ PEI derivatives}

Chemical strategies previously optimised for lipid substitution on $b$ PEI were herein employed for the derivatization of $0.6,1.2$ and $2 \mathrm{kDa} b$ PEI with St, LA, $\alpha \mathrm{LA}, \operatorname{PrA}$ and AcA (Scheme 1) [17,19]; the reaction feed ratio and the resulting degree of substitution, as measured by ${ }^{1} \mathrm{H}-\mathrm{NMR}$, of the resulting polymers are reported in Table 1 . As expected, the degree of substitution increased by increasing the feed ratio for every lipid, to give an average yield between 0.54 and 0.56 for all the substituents except St and LA that displayed a significantly lower average yield of 0.37 and 0.38 respectively. Every $b$ PEI derivative was water soluble.

\section{DNA complexation ability of $b$ PEI derivatives and polyplex characterization}

The capacity of every polymer to bind and complex pDNA was evaluated by SYBR Green I-exclusion assay. SYBR Green I is a non-specific intercalating DNA dye with high fluorescence quantum yield and a strong fluorescence enhancement when bound to DNA that has been commonly used to investigate DNA complexation [20]. Representative complexation curves as a function of polymer:DNA ratio $(w / w)$ are shown in Fig. 1a (please see Supplementary information, Fig. S1 for the complexation curves of all the $b$ PEI derivatives) and Table 1 shows the $w / w$ required for $50 \%$ pDNA binding $\left(\mathrm{BC}_{50}\right)$.

All the complexation curves shared a similar sigmoidal decrease in fluorescence as the polymer/pDNA weight ratios were increased. Generally speaking, the complexation ability of $b$ PEI derivatives decreased while increasing the degree of substitution, as indicated by the higher $\mathrm{BC}_{50}$ that reached values higher than 2.5 for PEI2-St12, PEI2-LA9 and PEI2- $\alpha$ LA8. In addition, a general positive and statistically significant correlation was found between $\mathrm{BC}_{50}$ and the degree of substitution for the three $b$ PEI backbones (Fig. 1b, Pearson correlation, $p<0.05$ ). This could be explained by the loss of amines available for interaction with negatively charged nucleic acids, owing to amine substitution and to the steric hindrance of the grafted molecules [17]. Dispersity in $\mathrm{BC}_{50}$ values was noted at higher substitutions ( $>2$ hydrophobes per PEI) for $1.2 \mathrm{kDa} b$ PEI-based derivatives. It is likely that the nature of the hydrophobic moieties became important in this region, differentially affecting nucleic acid binding due to steric hindrance. 


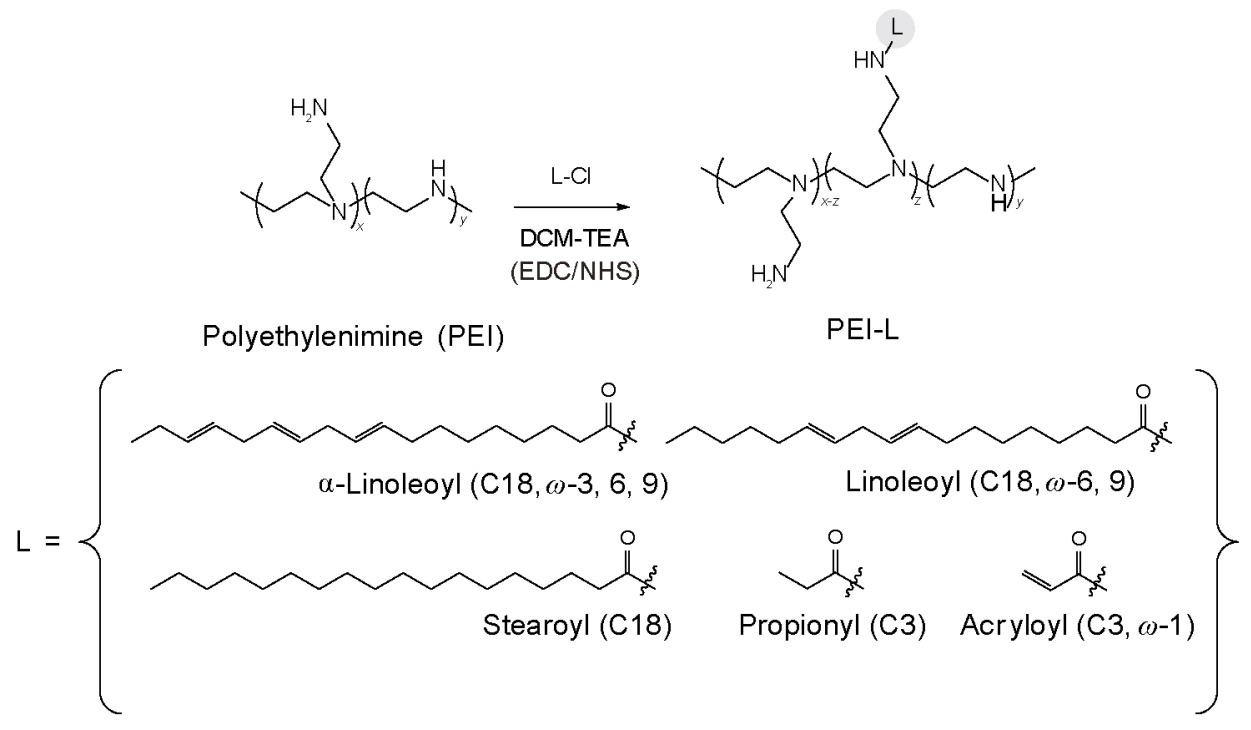

Scheme 1 Synthesis of hydrophobe-substituted $b$ PEI derivatives.
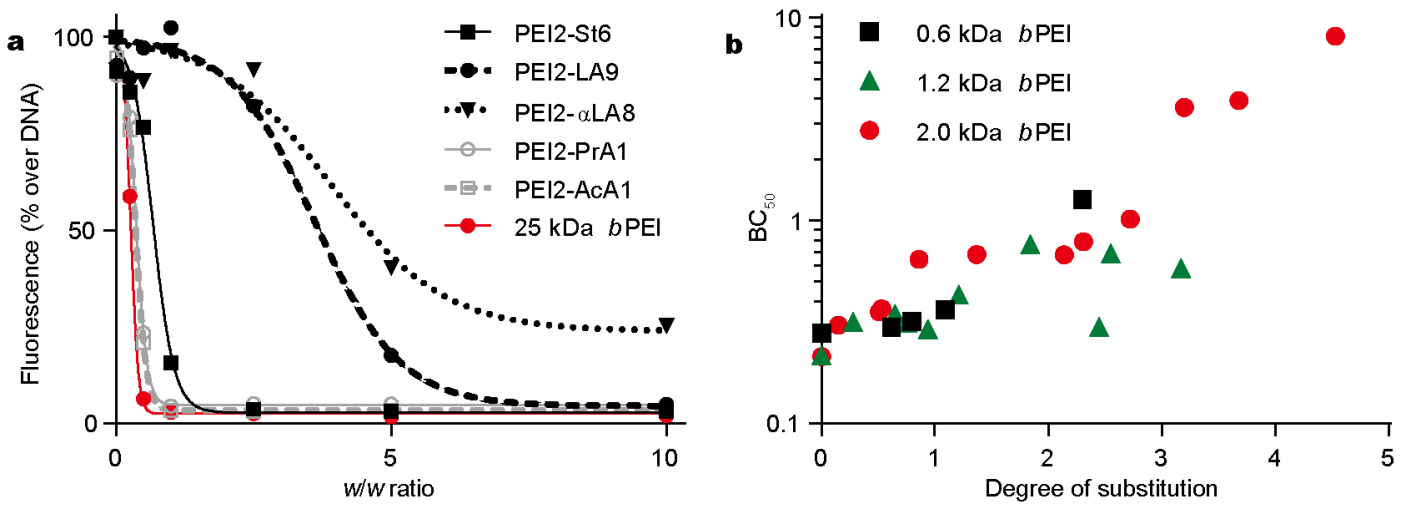

Figure 1 pDNA complexation ability of $b$ PEI derivatives as a function of polymer:DNA ratio $(w / w)$. (a) The complexation curves for PEI2-St6, PEI2LA9, PEI2- $\alpha$ LA8, PEI2-PrA1 and PEI2-AcA1 are reported as representative examples and compared with $25 \mathrm{kDa} b$ PEI. (b) BC 50 values as a function of the degree of substitution for 0.6 (black squares), 1.2 (green triangles) and $2.0 \mathrm{kDa} b \mathrm{PEI}$-based derivatives (red dots). A positive correlation was observed between the $\mathrm{BC}_{50}$ and degree of substitution, according to Pearson correlation $(r=0.91, p<0.05 ; r=0.67, p<0.05 ; r=0.84, p<0.05$ respectively for 0.6 , 1.2 and $2.0 \mathrm{kDa} b$ PEI-based derivatives).

As the physicochemical properties of polyplexes are expected to strongly affect their activity [26], the $\zeta_{\mathrm{P}}$ and $D_{\mathrm{H}}$ of polymer/pDNA complexes were measured at $w / w$ of 5 and 10, conditions that led to significant activity in transfection experiments; the results for the selected derivatives (the most efficient ones in transfection experiments) are shown in Table 2, while those relative to every $b$ PEI derivative are reported in the Supplementary information (Table $\mathrm{S} 1)$. For all the $b$ PEI derivatives, nanometric sizes were observed, with $D_{\mathrm{H}}$ always smaller than $300 \mathrm{~nm}$ and in general comparable to native $b$ PEIs, except for $0.6 \mathrm{kDa} b$ PEI that yielded relatively large particles bigger than $1 \mu \mathrm{m}$. The only exception was PEI2-St12 that already showed the lowest ability to complex $\mathrm{DNA}\left(\mathrm{BC}_{50}=8.088\right)$ and formed par- ticles larger than $1 \mu \mathrm{m}$ at $w / w 5$ and of ca. $700 \mu \mathrm{m}$ at $w / w$ 10. $\zeta_{\mathrm{P}}$ was always positive and between $+10 \mathrm{mV}$ and +30 $\mathrm{mV}$ at both $w / w$ tested, except for PEI1.2-LA6 that showed an almost neutral surface charge at $w / w 5$. In contrast to $\mathrm{BC}_{50}$, no significant correlation with the degree of substitution was observed for both size and $\zeta_{\mathrm{P}}$ (Pearson correlation, $p>0.05)$.

\section{Transfection of VSMCs by $\boldsymbol{b}$ PEI derivatives}

While the ability to efficiently complex DNA is necessary for gene delivery vectors, it does not guarantee effectiveness in transfection. Therefore, we tested the transfection efficiency and the cytotoxicity of polyplexes prepared at different $w / w$ with $b$ PEI derivatives on primary VSMCs 
Table 2 Hydrodynamic diameter $\left(D_{H}\right)$, polydispersity index (PDI) and $\zeta$-potential $\left(\zeta_{P}\right)$ of the polyplexes prepared using PEI1.2-LA6, PEI1.2- $\alpha$ LA2, PEI2-PrA2 and PEI1.2-AcA2 derivatives and of native $b$ PEIs $(0.6,1.2,2$ and $25 \mathrm{kDa})$ at $w / w 5$ and 10 and measured by DLS and laser Doppler microelectrophoresis

\begin{tabular}{|c|c|c|c|c|c|c|c|}
\hline Polymer & $w / w$ & $D_{H}(\mathrm{~nm})$ & St. Dev. $D_{H}(\mathrm{~nm})$ & PDI & St. Dev. PDI & $\zeta_{P}(\mathrm{mV})$ & St. Dev. $\zeta_{P}(\mathrm{mV})$ \\
\hline \multirow{2}{*}{ PEI1.2-LA6 } & 5 & 101 & 16 & 0.30 & 0.11 & -1.4 & 1.8 \\
\hline & 10 & 135 & 53 & 0.40 & 0.15 & 15.3 & 4.0 \\
\hline \multirow{2}{*}{ PEI1.2- $\alpha$ LA2 } & 5 & 97 & 25 & 0.33 & 0.09 & 21.0 & 1.5 \\
\hline & 10 & 229 & 10 & 0.54 & 0.01 & 23.1 & 0.8 \\
\hline \multirow{2}{*}{ PEI2-PrA1 } & 5 & 98 & 3 & 0.31 & 0.02 & 32.2 & 0.6 \\
\hline & 10 & 112 & 53 & 0.41 & 0.23 & 27.5 & 3.7 \\
\hline \multirow{2}{*}{ PEI1.2-AcA2 } & 5 & 104 & 3 & 0.36 & 0.04 & 26.0 & 6.1 \\
\hline & 10 & 94 & 7 & 0.21 & 0.12 & 22.8 & 4.5 \\
\hline \multirow{2}{*}{$0.6 \mathrm{kDa} b \mathrm{PEI}$} & 5 & 1381 & 281 & 1.00 & 0.00 & 14.4 & 5.2 \\
\hline & 10 & 1885 & 1715 & 0.87 & 0.13 & 15.6 & 0.2 \\
\hline $1.2 \mathrm{kDa} b \mathrm{PEI}$ & 5 & 139 & 7 & 0.45 & 0.03 & 28.9 & 0.6 \\
\hline \multirow{2}{*}{$2 \mathrm{kDa} b \mathrm{PEI}$} & 5 & 115 & 13 & 0.27 & 0.05 & 29.1 & 5.4 \\
\hline & 10 & 179 & 152 & 0.39 & 0.23 & 22.0 & 8.0 \\
\hline \multirow{2}{*}{$25 \mathrm{kDa} b \mathrm{PEI}$} & 5 & 112 & 20 & 0.35 & 0.05 & 32.8 & 0.7 \\
\hline & 10 & 104 & 16 & 0.29 & 0.09 & 28.2 & 0.9 \\
\hline
\end{tabular}

obtained from the human umbilical artery (HUASMCs) and from the porcine aorta (PAoSMCs), since pigs are commonly used in vascular research as a model of human disease [27]. The presence of serum in culture medium is necessary for long-term cell cultures and it is commonly employed to evaluate resistance to serum of gene delivery vectors prior to in vivo tests [28], consequently, transfection experiments were carried out in cDMEM, containing $10 \%$ FBS. Three $w / w$ ratios $(2.5,5$ and 10$)$ were tested, given the recognised dependence of transfection activity on this parameter. Culture medium containing polyplexes was replaced with fresh new cDMEM $4 \mathrm{~h}$ after transfection and 44 $\mathrm{h}$ later, transfection efficiency and cytotoxicity were evaluated. Firefly luciferase was used as a luciferase reporter system (pGL3), enabling a highly sensitive evaluation of transgene expression. It is worth to note that transfection experiments on HUASMCs were performed with a lower dose of polyplexes (80 vs.160 ng of pDNA/well) to avoid massive cytotoxicity.

Transfection outcomes on primary PAoSMCs and HUASMCs are shown in Figs 2 and 3 respectively. As expected, a strong dependence on polymer/DNA $w / w$ ratio was observed. A low $w / w$ of 2.5 always led to non-relevant transgene expression (data not shown) while $w / w 10$ was usually the most effective ratio for $b$ PEI derivatives. The $b$ PEI derivatives synthesised with $0.6 \mathrm{kDa} b$ PEI backbone were generally ineffective in transfecting VSMCs in com- parison to their counterparts synthesised from 1.2 or $2 \mathrm{kDa}$ $b$ PEI, suggesting that the substitution with hydrophobic moieties of $b$ PEI featuring too small polymeric backbone $(0.6 \mathrm{kDa})$ was ineffective in producing efficient gene vectors, even if such polymers did complex DNA effectively. No obvious general differences between the 1.2 and 2.0 $\mathrm{kDa} b$ PEI backbones could be observed both in terms of transfection efficiency and cytotoxicity, suggesting that both polymers were suitable substrates for hydrophobic substitution.

As expected from DNA complexation results, and as often reported for $b$ PEI derivatives [29-31], the degree of substitution strongly influenced the transfection properties. Very high substitution degrees led to low efficiency (PEI2-St12, PEI2-LA9, PEI1.2-aLA6) both in PAoSMCs and HUASMCs. Surprisingly, despite its low DNA complexation ability $\left(\mathrm{BC}_{50}=3.9\right)$, PEI2- $\alpha \mathrm{LA} 8$ displayed high transfection levels on PAoSMCs, comparable to its counterparts featuring a lower substitution degree, but negligible activity on HUASMCs. However, no significant direct correlation between transfection efficiency and the extent of lipid substitution was observed for the three $b$ PEI backbones (Fig. S2, Pearson correlation, $p>0.05$ ). This was quite expected since, as already observed in similar systems [16,19], increasing hydrophobic-moiety degree of substitution initially improves transfection until the attainment of an optimum after which efficiency decreases 

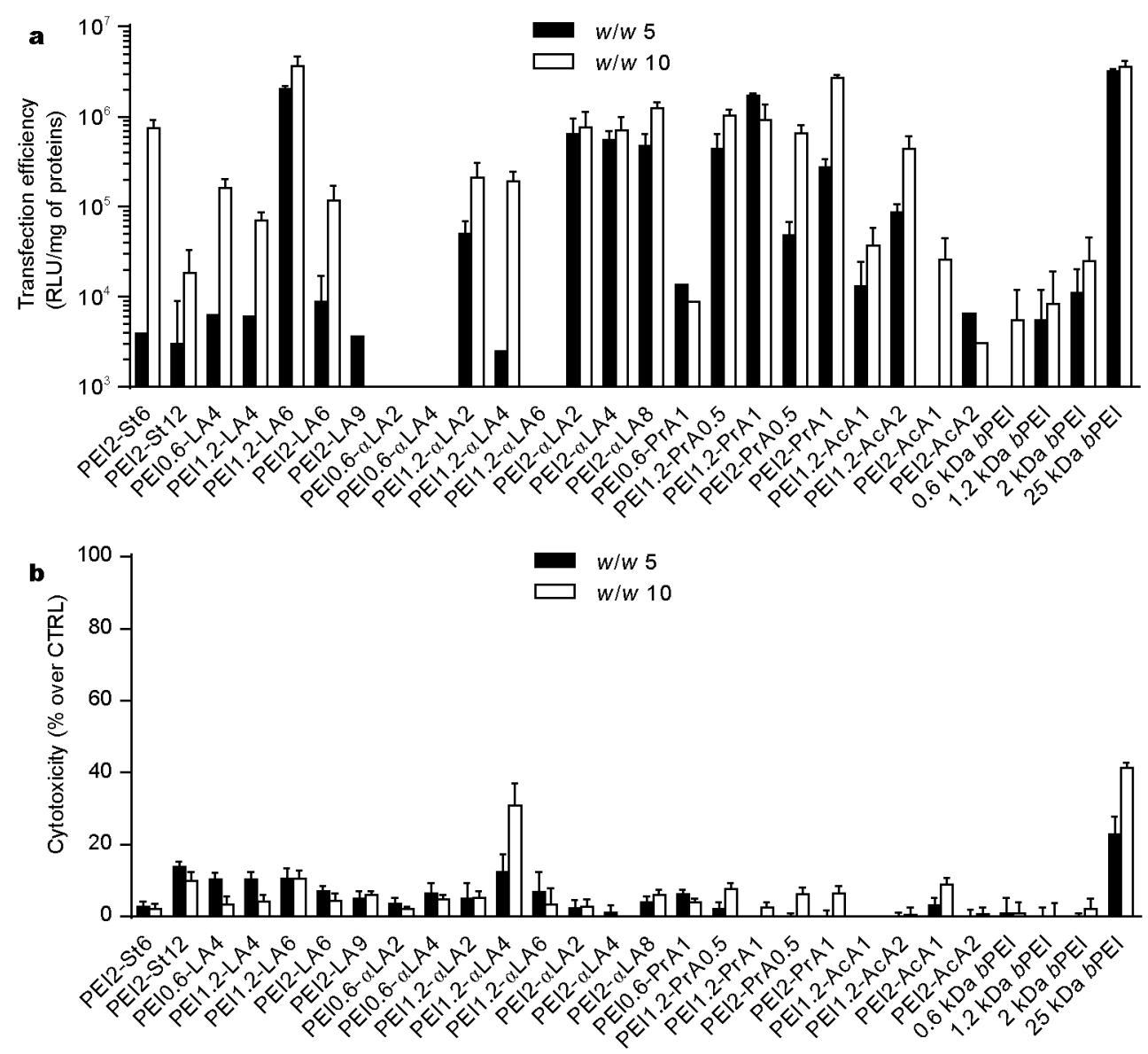

Figure 2 (a) Transfection efficiency and (b) cytotoxicity of $b$ PEI derivatives at $w / w 5$ and 10 on PAoSMCs. Cells were transfected with pGL3 and transfection efficiency was evaluated $48 \mathrm{~h}$ post-transfection and expressed as relative luminescence units (RLU) normalized over the total protein content in every cell lysate. Cytotoxicity was evaluated by AlamarBlue assay and expressed as percent viability loss with respect to untreated control cells. Missing bars in (a) indicate that no significant luciferase activity was detected. Results are shown as mean \pm standard deviation $(n \geq 4)$.

due to the excessive reduction of the number of amines available for DNA condensation.

The cytotoxicity of the polymers was generally lower than $20 \%$ on PAoSMCs, and lower than that of $25 \mathrm{kDa}$ $b$ PEI $(p<0.05)$ for all the derivatives tested, except for PEI1.2- $\alpha$ LA4, which was as toxic as $25 \mathrm{kDa} b$ PEI. In contrast, HUASMCs were much more sensitive to the overall cytotoxicity of transfectants and we thus reduced the polyplex dose delivered to avoid extensive cell death. As often observed in literature [32], derivatives with negligible activity exerted the lowest cytotoxicity and higher transfection efficiency levels were often associated with increased toxicity. A significant positive correlation between transfection efficiency and cytotoxicity was observed on PAoSMCs (Fig. S3a, Pearson correlation $r=0.49, p<0.05$ ) but not on HUASMC (Fig. S3b, Pearson correlation $r=$ $0.15, p>0.05$ ) even if both cytotoxicity (Fig. S4a, Pearson correlation $r=0.72, p<0.001)$ and transfection efficiency
(Fig. S4b, Pearson correlation $r=0.58, p<0.01$ ) significantly correlated between PAoSMCs and HUASMCs.

Even though St-substituted $b$ PEIs have been previously described as good transfection agents for siRNA delivery [13], our results showed that PEI-St polymers herein investigated were not so effective on both the VSMC types. PEI1.2-LA6 used at $w / w 10$ was the most effective PEI-LA derivative, as efficient as $(p>0.05)$ but much less cytotoxic (cytotoxicity: $10.4 \pm 2.4 \%$ vs. $22.9 \pm 4.7 \%$, PEI1.2-LA6 $w / w$ $10 v s .25 \mathrm{kDa}$ bPEI $w / w 5, p<0.05)$ than the gold standard polymeric transfectant $25 \mathrm{kDa} b$ PEI on PAoSMCs. PEI1.2LA6 was still the best LA-based derivative on HUASMCs but it was less efficient than $25 \mathrm{kDa} b$ PEI $(p<0.05)$ even if less cytotoxic (cytotoxicity: $40.8 \pm 3.1 \%$ vs. $50.2 \pm 2.9 \%$, PEI1.2-LA6 $w / w 10 v s .25 \mathrm{kDa} b$ PEI $w / w 5, p<0.05)$. Interestingly, LA-substituted $2 \mathrm{kDa} b \mathrm{PEI}$, already reported as one of the best $b$ PEI-lipid derivatives on 293T cells and rBMSC [17], did not show high transfection levels on 

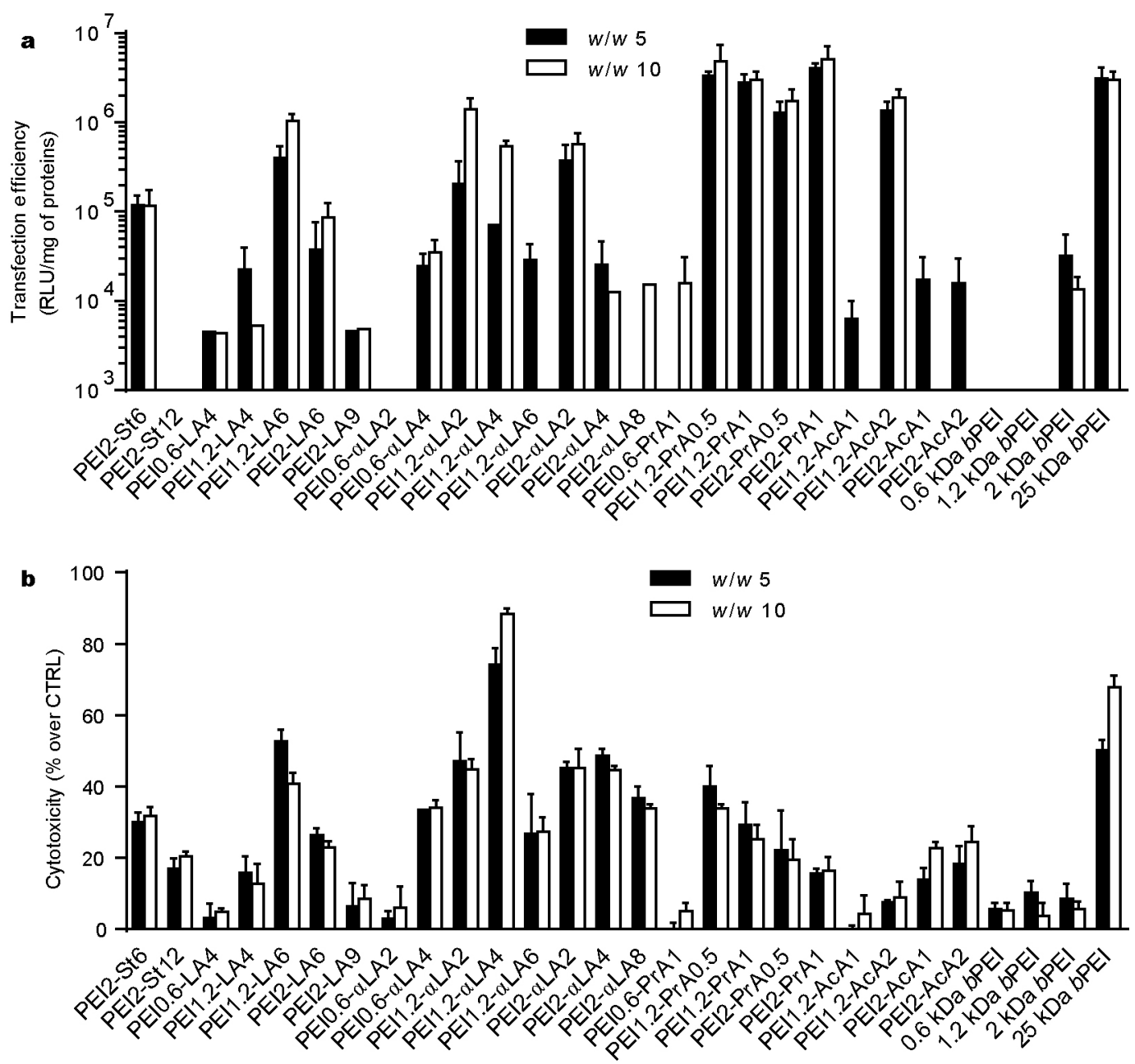

Figure 3 (a) Transfection efficiency and (b) cytotoxicity of $b$ PEI derivatives at $w / w 5$ and 10 on HUASMCs. Cells were transfected with pGL3 and transfection efficiency was measured $48 \mathrm{~h}$ post-transfection and expressed as RLU normalized over the total protein content in cell lysate. Cytotoxicity was evaluated by AlamarBlue assay and expressed as percent viability loss with respect to untreated control cells. Missing bars in (a) indicate that no significant luciferase activity was detected. Results are shown as mean \pm standard deviation $(n \geq 4)$.

VSMCs. These results suggest that the simultaneous fine tuning of the polymeric backbone MW and of the degree of substitution is a very important cell-specific determinant but that the optimized chemical properties are not universal for all cell types.

In line with the results previously reported on MDA231 and MCF-7 human breast cancer cells [19], PEI-PrA derivatives displayed high transgene expression especially on HUASMCs. Among them, PEI2-PrA1 was the most effective because the best compromise between high efficiency and low toxicity. In fact, PEI2-PrA1 derivative was as efficient as $(p>0.05)$ but much less cytotoxic than $25 \mathrm{kDa}$ $b$ PEI (cytotoxicity: $6.3 \pm 2.0 \%$ vs. $22.9 \pm 4.7 \%$, PEI2-PrA $w / w 10$ vs. $25 \mathrm{kDa} b$ PEI $w / w 5, p<0.05)$ on PAoSMCs. It was also the most effective among the tested derivatives on HUASMCs, with transfection levels more than twice higher than $25 \mathrm{kDa} b \mathrm{PEI}(p<0.05)$ and with significantly lower toxicity (cytotoxicity: $16.4 \pm 3.8 \% v s .50 .2 \pm 2.9 \%$, PEI2-PrA1 $w / w 10 v s .25 \mathrm{kDa}$ bPEI $w / w 5, p<0.05)$.

It is worthy of note that an increase in the unsaturation level of the substitute ( $\alpha \mathrm{LA}$ vs. LA, and AcA vs. PrA) did not lead to an improvement of the transfection activity of the polymers. PEI- $\alpha$ LA and PEI-AcA were invariably less efficient than PEI1.2-LA6 and PEI2-PrA1, respectively, on PAoSMCs $(p<0.05)$. Only PEI1.2- $\alpha$ LA2 displayed levels of transfection comparable to PEI1.2-LA6 $(p>0.05)$ on HUASMC, whilst PEI1.2-AcA2, which was by far the most efficient reagent belonging to the PEI-AcA family, showed a three-time lower luciferase expression as compared to PEI2-PrA1. Noteworthy, considering that oleic acid-sub- 
stituted PEI (degree of unsaturation = 1) already demonstrated a much lower efficiency as nuclei acid delivery agent with respect to PEI-LA [33] and taking into account the results on PEI-St (saturated), PEI-LA (degree of unsaturation $=2$ ) and PEI- $\alpha$ LA (degree of unsaturation = 3), it can be speculated that a degree of unsaturation of 2 is optimal for the substitution of low MW PEIs with the C18 lipids.

In light of these results, PEI1.2-LA6, PEI1.2-aLA2, PEI2PrA2, and PEI1.2-AcA2 polymers were selected for further investigation.

\section{Effect of centrifugation on the transfection activity of $b P E I$ derivatives on VSMCs}

Centrifugation of polyplexes is a simple way to accelerate their settling onto the cell surface, thus immediately increasing their concentration in the proximity of the cells. This approach can improve cellular uptake and the final transfection efficiency [34]. With this in mind, we challenged VSMCs with every selected $b$ PEI derivative subjected to a 5 min-centrifugation at $500 \times$ g immediately after the addition of polyplexes to the cells. Based on the results reported hereinabove, $b \mathrm{PEI}$ derivatives were used at $w / w 10$, while $25 \mathrm{kDa} b \mathrm{PEI}$ at $w / w 5$. As expected, centrifugation led to a substantial increase of the transfection levels, at least 10 folds higher than non-centrifuged controls (Fig. 4). The enhancement due to centrifugation was polymer-specific in the case of PAoSMCs, but not for HUASMCs for reasons to be elucidated. Unfortunately, higher cytotoxicity was also associated to the increase in transfection efficiency $(p<0.05$, centrifuged $v s$. non-centrifuged, for every polymer), however, the cytotoxicity of $b$ PEI derivatives was still significantly lower than that of $25 \mathrm{kDa} b$ PEI. These results highlight the importance

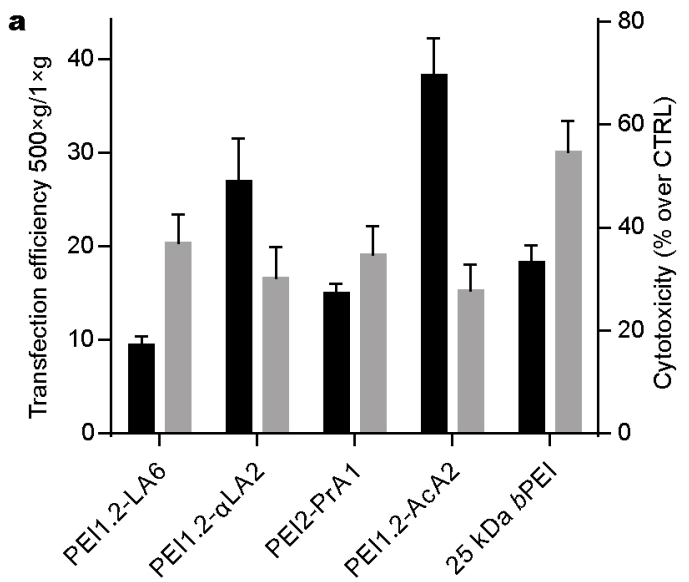

of sedimentation of gene delivery complexes on the cell surface to optimize transfection, but also strengthen the almost unavoidable relationship existing between increase in gene delivery activity and cytotoxicity.

\section{Transgene expression kinetics of $\boldsymbol{b P E I}$ derivatives in VSMCs}

The kinetics of transgene expression for the selected $b$ PEI derivatives was investigated to evaluate the medium- and long-term transfection efficiency on VSMCs. In accordance with the above results, polyplexes were centrifuged on the cells in order to maximise transfection efficiency. The plasmid encoding for the secreted Gaussia luciferase (pGLuc) was used for these studies. This enzyme endowed with chemiluminescent activity is constitutively secreted into the cell culture media that is harvested, allowing for real-time cell monitoring of the reporter gene expression and making easy the measurement of the expression kinetics.

As shown in Fig. 5a and b, a much more sustained transgene expression was generally observed on PAoSMCs with respect to HUASMCs. In the former, $25 \mathrm{kDa} b$ PEI showed a peak of expression within the first $48 \mathrm{~h}$ and then abruptly declined to levels nearly two orders of magnitude lower at day 10. However, for the bPEI derivatives, GLuc expression was stable or even increased over the first 4 days post-transfection, thereafter gradually decreasing by one order of magnitude. PEI1.2-LA6 and PEI2-PrA1 were the best performing derivatives. PEI2-PrA1 showed transfection levels comparable to $25 \mathrm{kDa} b$ PEI within the first 4 days and more than 5 -fold greater for longer times $(p<0.05$, PEI2-PrA1 $w / w 10$ s. $25 \mathrm{kDa} b \mathrm{PEI} w / w 5$ at day 10). The improved transfection efficiency over the long run was presumably due to the higher compatibility of low MW PEIs

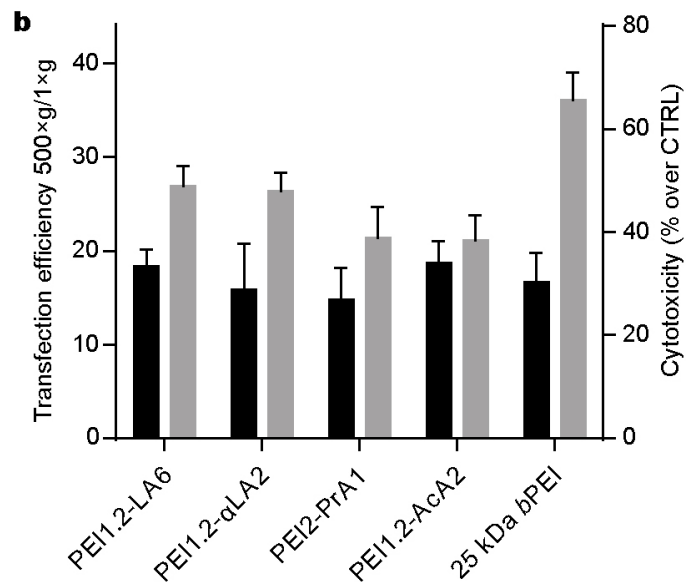

Figure 4 Effect of centrifugation on transfection by $b$ PEI derivatives on (a) PAoSMCs and (b) HUASMCs. The ratio between transfection efficiency obtained with $(500 \times \mathrm{g})$ and without $(1 \times \mathrm{g})$ centrifugation is reported (black bars). Cytotoxicity of centrifuged polyplexes (grey bars) is expressed as toxicity percent relative to untreated control cells. Results are shown as mean \pm standard deviation $(n \geq 4)$. 

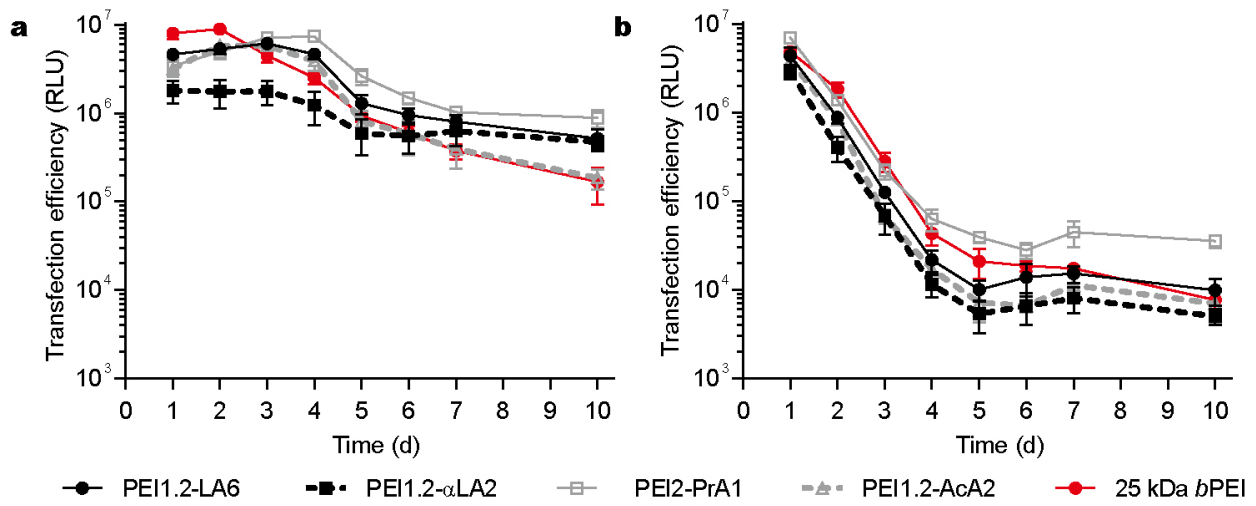

Figure 5 Kinetics of transfection efficiency of selected $b$ PEI derivatives on (a) PAoSMCs and (b) HUASMCs. Cells were transfected with pGLuc ( $w / w$ 10 for $b$ PEI derivatives, $w / w 5$ for $25 \mathrm{kDa} b \mathrm{PEI}$ ) and transfection efficiency was measured at different time points and expressed as RLU. Results are shown as mean \pm standard deviation $(n \geq 4)$.

(i.e., the higher percentage of modified cells remaining viable over a long period). In fact, the higher cytotoxicity of $25 \mathrm{kDa} b$ PEI is expected to negatively affect the long-term survival of the pool of cells that were actually transfected, leading to a more prominent decrease of exogenous transgene expression.

A common behaviour was observed for every polymer tested on HUASMC (Fig. 5b), with the expression maximum occurring at $24 \mathrm{~h}$ and a sharp decrease leading to quite steady levels of expression (at least two order of magnitude lower than day 1) after day 4. Of note, PEI2-PrA1 was again the most promising transfectant: transfection efficiency was comparable to that of $25 \mathrm{kDa} b \mathrm{PEI}$ within the first $72 \mathrm{~h}$, but superior over the long term (4.6 fold higher values at day 10; $p<0.05$, PEI2-PrA1 $w / w 10$ vs. $25 \mathrm{kDa}$ $b$ PEI $w / w 5$ at day 10$)$. Altogether, the superior gene expression profiles combined with the lower cytotoxicity with respect to the gold standard $25 \mathrm{kDa} b$ PEI make PEI2-PrA1 a very promising candidate for the transfection of VSMCs. These transfection results are extremely important because they were obtained in the presence of serum and upon centrifugation of the polyplexes over the cells, mimicking the high concentration at the cell surface that could be found in cardiovascular devices such as gene-eluting stents [35] or catheter balloons $[36,37]$.

\section{Cytotoxicity and transgene expression kinetics in HUVECs}

VSMCs are not the sole cell type playing a role in in-stent and post-angioplasty restenosis. ECs are present on the luminal surface of arteries and represent a suitable therapeutic target to prevent intima hyperplasia by speeding up the formation of a functional endothelium and preventing VSMCs migration and proliferation $[38,39]$. With this in mind, the two most promising $b$ PEI derivatives PEI1.2-
LA6 and PEI2-PrA1, were used to transfect primary HUVECs and compared to $25 \mathrm{kDa} b \mathrm{PEI}$. The optimised transfection conditions identified with VSMCs were employed and polyplexes were forced to sediment onto cells by centrifugation. A common behaviour in the transgene expression kinetics for every transfectant was apparent, with a maximum expression at day 1 , followed by a sharp decrease over time (Fig. 6a). In line with the results obtained on VSMCs, PEI2-PrA1 displayed an improved and long-lasting transfection in combination with cytotoxicity levels once again lower than $25 \mathrm{kDa} b \mathrm{PEI}$ (cytotoxicity: 30.6 $\pm 6.8 \%$ vs. $48.0 \pm 9.6 \%$, PEI2-PrA1 $w / w 10$ vs. $25 \mathrm{kDa}$ bPEI $w / w 5, p<0.05)$ and it can be thus considered as the most promising hydrophobe- $b$ PEI derivative for the transfection of vascular cells. It is worth noting that, even if PEI1.2-LA6 showed high efficiency on VSMC, it was much less effective on HUVECs compared to both PEI2-PrA1 and $25 \mathrm{kDa}$ $b$ PEI. Due to its specificity towards VSMCs and in consideration of its quite low cytotoxicity, PEI1.2-LA6 could therefore be proposed for the specific targeting of VSMCs in the vascular wall.

\section{CONCLUSIONS}

Herein, a library of hydrophobe-substituted low MW $b$ PEI derivatives with different $b$ PEI backbone MWs, degrees of substitution and types of hydrophobic substitutes were investigated as gene delivery vectors for primary VSMCs and ECs. The developed $b$ PEI derivatives were found effective in complexing DNA and, of note, the complexation ability was inversely correlated with the degree of substitution. Such polymers were generally less cytotoxic than the gold standard polymeric transfection reagent $25 \mathrm{kDa} b \mathrm{PEI}$ and some of them displayed an equal or even higher transfection efficiency. Very small polymers (i.e., $0.6 \mathrm{kDa} b$ PEI- 

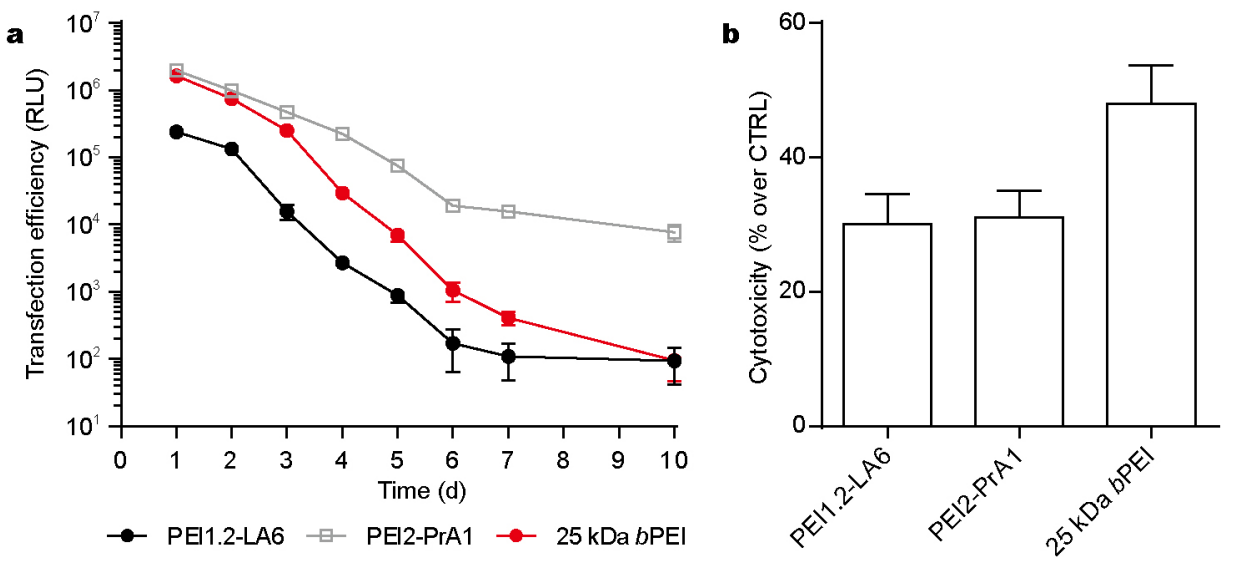

Figure 6 (a) Kinetics of transfection efficiency of selected $b$ PEI derivatives on HUVECs. Cells were transfected with pGLuc ( $w / w 10$ for $b$ PEI derivatives, $w / w 5$ for $25 \mathrm{kDa} b \mathrm{PEI}$ ) and transfection efficiency was measured at different time points and expressed as RLU. (b) Cytotoxicity of $b$ PEI derivatives on HUVECs. Cytotoxicity was evaluated by AlamarBlue assay $48 \mathrm{~h}$ post-transfection and expressed as percent toxicity relative to untreated cells. Results are shown as mean \pm standard deviation $(\mathrm{n} \geq 4)$.

based derivatives) were ineffective in transfection, while among 1.2 and $2 \mathrm{kDa} b$ PEI-derivatives, the propionic acidsubstituted polymer PEI2-PrA1 was the most promising compound for VSMCs transfection. Due to the increase of polyplex sedimentation on the cell surface, centrifugation of polyplexes over the cells greatly improved transfection efficiency. It is worth noting that in this experimental setup, mimicking the high polyplex concentration occurring in cardiovascular devices such as gene-eluting stents or catheter balloons, the activity of PEI2-PrA1 was higher and more sustained than $25 \mathrm{kDa} b$ PEI on both VSMCs and ECs, while maintaining significantly lower cytotoxicity levels. Interestingly, the linoleic acid-substituted polymer PEI1.2-LA6 was at least as effective as and less cytotoxic than $25 \mathrm{kDa} b$ PEI on VSMCs but exerted low transfection on ECs, suggesting that this polymer could be used for the selective gene delivery to VSMCs in blood vessels. Altogether, in this work, hydrophobic moiety-substituted low MW $b$ PEI derivatives with superior transfection properties on vascular cells were identified, with possible applications in non-viral gene therapy approaches aimed at the targeted inhibition of vascular pathologies such as intimal hyperplasia and in-stent/post-angioplasty restenosis.

Received 13 March 2017; accepted 31 March 2017; published online 20 April 2017

1 Mozaffarian D, Benjamin EJ, Go AS, et al. Executive summary: heart disease and stroke statistics-2015 update: a report from the American Heart Association. Circulation, 2015, 131: 434-441

2 Shan L, Saxena A, McMahon R, et al. Coronary artery bypass graft surgery in the elderly: a review of postoperative quality of life. Circulation, 2013, 128: 2333-2343

3 Marx SO, Totary-Jain H, Marks AR. Vascular smooth muscle cell proliferation in restenosis. Circ-Cardiovasc Interv, 2011, 4: 104-111

4 Bauters C, Isner J. The biology of restenosis. Prog Cardiovasc Dis, 1997, 40: 107-116

5 Arnold JD, Mountain DJH, Freeman MB, et al. Smooth muscle cell polymeric transfection is an efficient alternative to traditional methods of experimental gene therapy. J Surg Res, 2012, 177: 178-184

6 Fang YL, Chen XG, Godbey WT. Gene delivery in tissue engineering and regenerative medicine. J Biomed Mater Res, 2015, 103: 1679-1699

7 Dean DA. Nonviral gene transfer to skeletal, smooth, and cardiac muscle in living animals. AJP-Cell Physiol, 2005, 289: C233-C245

8 Pezzoli D, Chiesa R, De Nardo L, et al. We still have a long way to go to effectively deliver genes! J Appl Biomater Funct Mater, 2012, 2: $82-91$

9 Pezzoli D, Candiani G. Non-viral gene delivery strategies for gene therapy: a "ménage à trois" among nucleic acids, materials, and the biological environment. J Nanopart Res, 2013, 15: 1523

10 Akinc A, Thomas M, Klibanov AM, et al. Exploring polyethylenimine-mediated DNA transfection and the proton sponge hypothesis. J Gene Med, 2005, 7: 657-663

11 Mintzer MA, Simanek EE. Nonviral vectors for gene delivery. Chem Rev, 2009, 109: 259-302

12 Pezzoli D, Olimpieri F, Malloggi C, et al. Chitosan-graft-branched polyethylenimine copolymers: influence of degree of grafting on transfection behavior. PLoS ONE, 2012, 7: e34711

13 Alshamsan A, Haddadi A, Incani V, et al. Formulation and delivery of siRNA by oleic acid and stearic acid modified polyethylenimine. Mol Pharm, 2009, 6: 121-133

14 Han S, Mahato RI, Kim SW. Water-soluble lipopolymer for gene delivery. Bioconjugate Chem, 2001, 12: 337-345

15 Thomas M, Klibanov AM. Enhancing polyethylenimine's delivery of plasmid DNA into mammalian cells. Proc Natl Acad Sci USA, 2002, 99: 14640-14645

16 Zheng M, Zhong Y, Meng F, et al. Lipoic acid modified low molecular weight polyethylenimine mediates nontoxic and highly potent in vitro gene transfection. Mol Pharm, 2011, 8: 2434-2443

17 Neamnark A, Suwantong O, Bahadur RKC, et al. Aliphatic lipid substitution on $2 \mathrm{kD}$ a polyethylenimine improves plasmid delivery 
and transgene expression. Mol Pharm, 2009, 6: 1798-1815

18

Bahadur KCR, Landry B, Aliabadi HM, et al. Lipid substitution on low molecular weight $(0.6-2.0 \mathrm{kDa})$ polyethylenimine leads to a higher zeta potential of plasmid DNA and enhances transgene expression. Acta Biomater, 2011, 7: 2209-2217

19 Thapa B, Plianwong S, Remant Bahadur KC, et al. Small hydrophobe substitution on polyethylenimine for plasmid DNA delivery: optimal substitution is critical for effective delivery. Acta Biomater, 2016, 33: 213-224

20 D'Andrea C, Pezzoli D, Malloggi C, et al. The study of polyplex formation and stability by time-resolved fluorescence spectroscopy of SYBR Green I-stained DNA. Photochem Photobiol Sci, 2014, 13: 1680-1689

21 Pezzoli D, Kajaste-Rudnitski A, Chiesa R, et al. Lipid-based nanoparticles as nonviral gene delivery vectors. In: Bergese P, Hamad-Schifferli K (eds.). Nanomaterial Interfaces in Biology: Methods and Protocols, Methods in Molecular Biology. New York: Humana Press, 2013, 1025: 269-279

22 Chang KH, Park JM, Lee MY. Feasibility of simultaneous measurement of cytosolic calcium and hydrogen peroxide in vascular smooth muscle cells. BMB Rep, 2013, 46: 600-605

23 Fan Z, Chen D, Deng CX. Improving ultrasound gene transfection efficiency by controlling ultrasound excitation of microbubbles. J Control Release, 2013, 170: 401-413

24 Gresch O, Altrogge L. Transfection of difficult-to-transfect primary mammalian cells. In: Hartley JL (ed.). Protein Expression in Mammalian Cells: Methods and Protocols, Methods in Molecular Biology. New York: Humana Press, 2012, 801: 65-74

25 Hsu CYM, Hendzel M, Uludağ H. Improved transfection efficiency of an aliphatic lipid substituted $2 \mathrm{kDa}$ polyethylenimine is attributed to enhanced nuclear association and uptake in rat bone marrow stromal cell. J Gene Med, 2011, 13: 46-59

26 Pezzoli D, Giupponi E, Mantovani D, et al. Size matters for in vitro gene delivery: investigating the relationships among complexation protocol, transfection medium, size and sedimentation. Sci Rep, 2017, 7: 44134

27 Suzuki Y, Yeung AC, Ikeno F. The representative porcine model for human cardiovascular disease. J Biomed Biotech, 2011, 2011: 1-10

28 Malloggi C, Pezzoli D, Magagnin L, et al. Comparative evaluation and optimization of off-the-shelf cationic polymers for gene delivery purposes. Polym Chem, 2015, 6: 6325-6339

29 Dube B, Rose L, Sawant K, et al. Cholic acid modified 2 kDa polyethylenimine as efficient transfection agent. Biotechnol Prog, 2013, 29: 1337-1341

30 Zhang QF, Luan CR, Yin DX, et al. Amino acid-modified polyethylenimines with enhanced gene delivery efficiency and biocompatibility. Polymers, 2015, 7: 2316-2331

31 Pezzoli D, Tarsini P, Melone L, et al. RGD-derivatized PEI-PEG copolymers: influence of the degree of substitution on the targeting behavior. J Drug Deliver Sci Tech, 2017, 37: 115-122

32 Breunig M, Lungwitz U, Liebl R, et al. Breaking up the correlation between efficacy and toxicity for nonviral gene delivery. Proc Natl Acad Sci USA, 2007, 104: 14454-14459

33 Aliabadi HM, Landry B, Bahadur RK, et al. Impact of lipid substitution on assembly and delivery of siRNA by cationic polymers. Macromol Biosci, 2011, 11: 662-672

34 Hsu CYM, Uludağ H. A simple and rapid nonviral approach to efficiently transfect primary tissue-derived cells using polyethylenimine. Nat Protoc, 2012, 7: 935-945

35 Fishbein I, Chorny M, Adamo RF, et al. Endovascular gene delivery from a stent platform: gene- eluting stents. Angiol, 2013, 1: 1000109

36 Saurer EM, Yamanouchi D, Liu B, et al. Delivery of plasmid DNA to vascular tissue in vivo using catheter balloons coated with polyelectrolyte multilayers. Biomaterials, 2011, 32: 610-618

37 Riessen R, Rahimizadeh H, Blessing E, et al. Arterial gene transfer using pure DNA applied directly to a hydrogel-coated angioplasty balloon. Hum Gene Ther, 1993, 4: 749-758

38 Sharif F, Hynes SO, Cooney R, et al. Gene-eluting stents: adenovirus-mediated delivery of eNOS to the blood vessel wall accelerates re-endothelialization and inhibits restenosis. Mol Therapy, 2008, 16: 1674-1680

39 Patel SD, Waltham M, Wadoodi A, et al. The role of endothelial cells and their progenitors in intimal hyperplasia. Therapeutic Adv Cardiovascular Disease, 2010, 4: 129-141

Acknowledgments Pezzoli D and Tsekoura EK were awarded a postdoctoral and doctoral scholarship, respectively, from the NSERC CREATE Program in Regenerative Medicine, www.ncprm.ulaval.ca. The studies were financially supported by the Natural Science and Engineering Research Council of Canada, (Discovery Grant to Uludağ H and Mantovani D), the Canadian Institute for Health Research (Operating grant to Uludağ $\mathrm{H}$ ), and the Fonds de Recherche du Quebec sur les Natures et Technologies (Bilateral Grant to Mantovani D). We thank Dr. Vishwa Somayaji for ${ }^{1} \mathrm{H}-\mathrm{NMR}$ analysis of the polymer samples

Author contributions Pezzoli D, Uludağ H, Mantovani D and Candiani G conceived the idea and designed the experiments; Pezzoli D, Tsekoura EK and Bahadur KC R performed the experiments; Pezzoli D and Uludağ $\mathrm{H}$ analysed the data and wrote the manuscript with support from Candiani G and Mantovani D. All authors contributed to the general discussion.

Conflict of interest Bahadur KCR and Uludağ $\mathrm{H}$ hold ownership position in RJH Biosciences Inc. intended to commercialise the described polymers.

Supplementary information Supplementary data are available in the online version of the paper. 

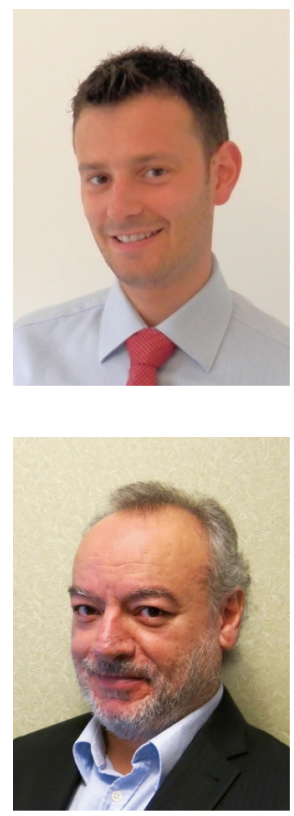

Daniele Pezzoli received his PhD degree in Materials Engineering at Politecnico di Milano (Milan, Italy) in 2011, under the supervision of Prof. Gabriele Candiani. He is currently a postdoctoral fellow in the Laboratory for Biomaterials and Bioengineering of Université Laval (Quebec City, Canada), led by Prof. Diego Mantovani. His current research is focused on vascular tissue engineering using collagen gel-based scaffolds and on the development of polymeric non-viral gene delivery systems for tissue engineering applications.

Hasan Uludağ has been with the University of Alberta since 1997, designing functional biomaterials to realize the therapeutic potential of nucleic acids. He obtained dual BSc degrees in Biomedical Engineering and Biology from Brown University (USA) in 1989. He then completed his PhD degree in 1993 at the Department of Chemical Engineering at the University of Toronto. He spent four years at Genetics Institute Inc. (USA), where he contributed to development of a bone-inducing BMP device.

\section{疏水基取代的分枝状聚乙酰亚胺衍生物：促进原发性血管细胞中的转染}

Daniele Pezzoli ${ }^{1 *}$, Eleni K. Tsekoura ${ }^{2}$, Remant Bahadur K.C. ${ }^{2}$, Gabriele Candiani ${ }^{3}$, Diego Mantovani ${ }^{1}$, Hasan Uludağ ${ }^{2 *}$

摘要 针对血管细胞的基因治疗代表了一种有望用于预防和治疗内膜增生、血管支架狭窄和血管成形术后狭窄等病理状态的方法. 聚合 物非病毒载体的基因传递系统可以安全替代病毒载体, 但是为了提高临床效果, 它们的治疗效率及细胞相容性还需要进一步改善. 本文合 成了一系列 24 种被疏水基团修饰的分枝状聚乙酰亚胺衍生物( $b$ PEI), 并进行了表征及在体外原发性血管细胞内的测试, 旨在笁选出具有优 异的转染效率和低细胞毒性的传递剂. 低分子量的聚乙酰亚胺(0.6, 1.2 and $2 \mathrm{kDa}$ ) 以不同取代程度接枝上了不同饱和度的长(C18)和短(C3) 的不饱和脂肪链. 丙酰取代衍生物(PEI2-PrA1, C3:0)在血管平滑肌细胞和内皮细胞转染中是最有效的, 与著名的黄金标准 $25 \mathrm{kDa} b \mathrm{PEI}$ 相比, 具有更优异、更持久的基因表达, 且毒性更低. 此外, 亚油酰基取代衍生物(PEI1.2-LA6, C18:2) 由于在血管平滑肌细胞转染过程中效率高, 而在内皮细胞中相对无效, 且其具有可容忍的细胞毒性, 可作为特定靶向于血管平滑肌细胞的载体. 